\title{
Nonclassical properties and algebraic characteristics of negative binomial states in quantized radiation fields
}

\author{
Xiao-Guang Wang* Shao-Hua Pan and Guo-Zhen Yang \\ CCAST(World Laboratory),P.O.Box 8730, Beijing 100080 \\ and Laboratory of Optical Physics, Institute of Physics, Chinese Academy of \\ Sciences,Beijing 100080, P.R.China
}

(July 23, 2018)

\begin{abstract}
We study the nonclassical properties and algebraic characteristics of the negative binomial states introduced by Barnett recently. The ladder operator formalism and displacement operator formalism of the negative binomial states are found and the algebra involved turns out to be the $\mathrm{SU}(1,1)$ Lie algebra via the generalized Holstein-Primarkoff realization. These states are essentially Peremolov's SU(1,1) coherent states. We reveal their connection with the geometric states and find that they are excited geometric states. As intermediate states, they interpolate between the number states and geometric states. We also point out that they can be recognized as the nonlinear coherent states. Their nonclassical properties, such as sub-Poissonian distribution and squeezing effect are discussed. The quasiprobability distributions
\end{abstract}

*email: xyw@aphy.iphy.ac.cn

Ref.number: D9123

Section: Optical Physics and Quantum Optics

Running title: Negative binomial states in quantized radiation fields

Accepted in EPJD on 22/11/99 
in phase space, namely the $\mathrm{Q}$ and Wigner functions, are studied in detail. We also propose two methods of generation of the negative binomial states.

PACS numbers:42.50.Dv,03.65.Db,32.80.Pj,42.50.Vk

Typeset using REVTEX 


\section{INTRODUCTION}

Since Stoler et al. introduced the binomial states (BSs)[1], the so-called intermediate states have attracted considerable attention of physicists in the field of quantum optics. A feature of these states is that they interpolate between two fundamental quantum states, such as the number, coherent and squeezed states, and reduce to them in two different limits. For instance, the BSs interpolate between the coherent states (the most classical) and the number states (the most nonclassical)[1-6], while the negative binomial states (NBSs) interpolate between the coherent states and geometric states[7-11]. Another feature of some intermediate states is that their photon number distributions are some famous discrete probability distributions in probability theory: the BS corresponds to the binomial distribution, the NBS to the negative binomial distribution, the hypergeometric state[12] to the hypergeometric distribution, and the negative hypergeometric state[13] to the negative hypergeometric distribution.

Recently Barnett introduced a new definition of NBS[14],

$$
\begin{aligned}
|\eta, M\rangle & =\sum_{n=M}^{\infty} C_{n}(\eta, M)|n\rangle \\
& =\sum_{n=M}^{\infty}\left[\left(\begin{array}{c}
n \\
M
\end{array}\right) \eta^{M+1}(1-\eta)^{n-M}\right]^{1 / 2}|n\rangle,
\end{aligned}
$$

where $|n\rangle$ is the usual number state, $0<\eta \leq 1$ and $M$ is a non-negative integer. They find that the NBS $|\eta, M\rangle$ and the BS have similar properties if the roles of the creation operator $a^{\dagger}$ and annihilation operator $a$ are interchanged. The photon number probability $\left|C_{n}(\eta, M)\right|^{2}$ is associated with the probability that $n$ photons were present given that $M$ are found and that the probability for successfully detecting any single photon is $\eta$. Mixed states with the photon number probability include those applicable to photodetection and optical amplification[15].

The BS is a intermediate number-coherent state and the original NBS is a intermediate geometric-coherent state. One question naturally arises that if there exist an intermediate state which interpolates between the number and geometric state. In reality, the new NBS 
is just the intermediate number-geometric state. This fact will be seen in the next section. Thus, we have three intermediate states which interpolate between two of the three fundamental states (the number, coherent and geometric states).

In the present paper we shall study the nonclassical properties and algebraic characteristics of the new NBS. The ladder operator formalism, displacement operator formalism and related algebraic structure will be formulated in section II. It is interesting that the algebraic structure is the $\mathrm{SU}(1,1)$ Lie algebra via the generalized Holstein-Primarkoff realization. In section III, we will show that the NBS can be viewed as excited geometric states, intermediate number-geometric states and nonlinear coherent states. The nonclassical properties, such as sub-Poissonian distribution and squeezing effect will be investigated in detail in section IV. The Q and Wigner functions are studied in Section V and the two methods of generation of the NBS are proposed in section VI. A conclusion is given in section VII.

\section{LADDER OPERATOR FORMALISM ,DISPLACEMENT OPERATOR FORMALISM AND ALGEBRAIC STRUCTURE OF THE NEW NBS}

\section{A. Ladder operator formalism and algebraic structure}

It is known that the BSs are special $\mathrm{SU}(2)$ coherent states $[5,6]$ and the original NBSs are Perelomov's SU(1,1) coherent states[11] via the standard Holstein-Primakoff realizations. So we expect that the algebra involved in the new NBS is $\mathrm{SU}(1,1)$ Lie algebra.

It is easy to evaluate that

$$
a^{\dagger n}|\eta, M\rangle=\left[\frac{(M+n) !}{M ! \eta^{n}}\right]^{1 / 2}|\eta, M+n\rangle .
$$

In particular, for $n=1$, we get

$$
a^{\dagger}|\eta, M\rangle=\left(\frac{M+1}{\eta}\right)^{1 / 2}|\eta, M+1\rangle .
$$

The creation operator raises the NBS $|\eta, M\rangle$ to $|\eta, M+1\rangle$. This property is similar to the action of the creation operator on the Fock state $|M\rangle$,

$$
a^{\dagger}|M\rangle=\sqrt{M+1}|M+1\rangle
$$


Actually, in the limit of $\eta \rightarrow 1$, the NBS $|\eta, M\rangle^{-}$reduces to the number state $|M\rangle$ and Eq.(3) naturally reduces to Eq.(4).

The key and interesting point is that there exists another operator $\sqrt{\hat{N}-M}$ which also raises the NBS $|\eta, M\rangle$ to $|\eta, M+1\rangle$. From Eq.(1), the following equation is directly derived as

$$
\sqrt{\hat{N}-M}|\eta, M\rangle=\left(\frac{1-\eta}{\eta}\right)^{1 / 2} \sqrt{M+1}|\eta, M+1\rangle
$$

Comparing Eq.(3) and Eq.(5), we get

$$
\sqrt{\hat{N}-M}|\eta, M\rangle=\sqrt{1-\eta} a^{\dagger}|\eta, M\rangle
$$

Multiplying the both sides of the above equation by the operator $\sqrt{\hat{N}-M}$ from left, we obtain the ladder operator formalism of the NBS as

$$
\left(\hat{N}-\sqrt{1-\eta} \sqrt{\hat{N}-M} a^{\dagger}\right)|\eta, M\rangle=M|\eta, M\rangle .
$$

In the limit of $\eta \rightarrow 1$, we find that the NBS $|\eta, M\rangle$ reduces to the number state $|M\rangle$ and Eq.(7) to the equation $\hat{N}|M\rangle=M|M\rangle$ as expected.

It can be proved that the operators appearing in Eq.(7) can form $\mathrm{SU}(1,1)$ Lie algebra:

$$
K_{0}=\hat{N}-\frac{M-1}{2}, K_{+}=\sqrt{\hat{N}-M} a^{\dagger}, K_{-}=a \sqrt{\hat{N}-M}
$$

Reminding of the standard Holstein-Primarkoff realization of $\mathrm{SU}(1,1)$ Lie algebra, $J_{0}=$ $\hat{N}+\frac{M}{2}, J_{+}=\sqrt{M+\hat{N}-1} a^{\dagger}, J_{-}=a \sqrt{M+\hat{N}-1}$, we call the new realization as generalized Holstein-Primakoff realization. To our knowledge, the new realization of SU(1,1) Lie algebra seems not to be addressed in the literature.

In terms of the generators $K_{0}, K_{+}$and $K_{-}$of the $\mathrm{SU}(1,1)$ Lie algebra, Eq.(7) is rewritten as

$$
\left(K_{0}-\sqrt{1-\eta} K_{+}\right)|\eta, M\rangle=\frac{M+1}{2}|\eta, M\rangle .
$$




\section{B. Displacement operator formalism}

Now we try to find the displacement operator formalism of the NBS. To this end, let us rewrite the NBS as

$$
\begin{aligned}
|\eta, M\rangle & =\eta^{\frac{M+1}{2}} \sum_{n=0}^{\infty}\left[\left(\begin{array}{c}
M+n \\
M
\end{array}\right)(1-\eta)^{n}\right]^{1 / 2}|M+n\rangle \\
& =\eta^{\frac{M+1}{2}} \sum_{n=0}^{\infty} \frac{\left(\sqrt{1-\eta} a^{\dagger}\right)^{n}}{\sqrt{n !}}|M\rangle .
\end{aligned}
$$

Then by making use of the following identity

$$
\left[f(\hat{N}) a^{\dagger}\right]^{n}=a^{\dagger n} f(\hat{N}+n) f(\hat{N}+n-1) \ldots f(\hat{N}+1)
$$

Eq.(10) can be written in the exponential form

$$
|\eta, M\rangle=\eta^{\frac{M+1}{2}} e^{\sqrt{1-\eta} K_{+}}|M\rangle
$$

Note that $K_{-}|M\rangle=0$, then the displacement operator formalism is obtained as

$$
\begin{aligned}
|\eta, M\rangle & =e^{\sqrt{1-\eta} K_{+}} \eta^{K_{0}} e^{-\sqrt{1-\eta} K_{-}}|M\rangle \\
& =e^{\xi\left(K_{+}-K_{-}\right)}|M\rangle
\end{aligned}
$$

where $\xi=\operatorname{arctanh} \sqrt{1-\eta}$. In the derivation of the above equation, we have used the identity

$$
e^{\alpha K_{+}-\alpha^{*} K_{-}}=e^{\gamma K_{+}}\left(1-|\gamma|^{2}\right)^{K_{0}} e^{-\gamma^{*} K_{-}},
$$

where $\gamma=\alpha \tanh |\alpha| /|\alpha|$. As seen from Eq.(13), the NBS can be simply recognized as SU(1,1) displaced number states. Actually, the NBS are essentially Peremolov's coherent states as shown below.

On the space

$$
S=\operatorname{span}\{|n+M\rangle \equiv|n ; k\rangle \mid n=0,1,2 \ldots\}, k=\frac{M+1}{2}
$$

we have

$$
\begin{aligned}
K_{+}|n ; k\rangle & =\sqrt{(n+1)(2 k+n)}|n+1 ; k\rangle \\
K_{-}|n ; k\rangle & =\sqrt{n(2 k+n-1)}|n-1 ; k\rangle \\
K_{0}|n ; k\rangle & =(n+k)|n ; k\rangle
\end{aligned}
$$


This is the discrete representation of $\mathrm{SU}(1,1)$ Lie algebra with Bargaman index $k=$ $(M+1) / 2$. We see that the generalized Holstein-Primakoff realization gives rise to the representation of $\mathrm{SU}(1,1)$ on the space $S$. Note that $|M\rangle=|0 ; k\rangle$, the NBS can be written as

$$
|\eta, M\rangle=e^{\xi\left(K_{+}-K_{-}\right)}|0 ; k\rangle .
$$

This shows the NBS are essentially Peremolov's coherent states.

\section{THE NBS AS EXCITED GEOMETRIC STATE, INTERMEDIATE NUMBER-GEOMETRIC STATE AND NONLINEAR COHERENT STATE}

\section{A. As excited geometric states and intermediate number-geometric states}

From Eq.(10) we obtain

$$
\begin{aligned}
|\eta, M\rangle & =\eta^{(M+1) / 2} \sum_{n=0}^{\infty}\left(\begin{array}{c}
M+n \\
M
\end{array}\right)^{1 / 2}(1-\eta)^{n / 2}|M+n\rangle \\
& =\frac{\eta^{(M+1) / 2}}{\sqrt{M !}} a^{\dagger M} \sum_{n=0}^{\infty}(1-\eta)^{n / 2}|n\rangle \\
& =\frac{\eta^{M / 2}}{\sqrt{M !}} a^{\dagger M}|\eta\rangle_{g},
\end{aligned}
$$

where

$$
|\eta\rangle_{g}=\eta^{1 / 2} \sum_{n=0}^{\infty}(1-\eta)^{n / 2}|n\rangle
$$

is the geometric state[16-21], which is also called Susskind-Glogower phase state[11], phase eigenstate[16,17], and coherent phase state[19]. The photon number distribution is $\eta(1-\eta)^{n}$, the geometric distribution. From Eq.(18) the NBSs can be generated by repeated application

of the creation operator $a^{\dagger}$ on the geometric states. This shows that the NBS belongs to an interesting class of nonclassical states, excited quantum states. These states are first introduced by Agarwal and Tara as excited coherent states[22]. So the NBSs can be viewed as excited geometric states. 
Setting $M=0$ in Eq.(18), we get

$$
|\eta, 0\rangle=|\eta\rangle_{g}
$$

which shows that the NBS reduces to the geometric states for $M=0$. Note that the NBS reduces to the number state $|M\rangle$ in the limit of $\eta \rightarrow 1$. Thus, the NBS interpolates between the number state and geometric state and can be viewed as number-geometric state.

\section{B. As nonlinear coherent states}

The geometric states are the eigenstates of the Susskind-Glogower phase operator[23] $(1+N)^{1 / 2} a$, obeying the equation

$$
(N+1)^{-1 / 2} a|\eta\rangle_{g}=\sqrt{1-\eta}|\eta\rangle_{g}
$$

Comparing with the definition of the nonlinear coherent states $|\alpha\rangle_{n l}[24,25]$

$$
f(N) a|\alpha\rangle_{n l}=\alpha|\alpha\rangle_{n l}
$$

we know that the geometric states are nonlinear coherent states with the nonlinear function $f(N)=(N+1)^{-1 / 2}$

In a previous work, we have proved a general result that the excited nonlinear coherent states are still nonlinear coherent states[26]. Since the geometric states are nonlinear coherent states and the NBSs can be recognized as excited geometric states, we infer that the NBSs are nonlinear coherent states. In fact, multiplying Eq.(7) by the annihilation operator $a$ from the left, we get

$$
(N+1-M) a|\eta, M\rangle=\sqrt{1-\eta} \sqrt{N+1-M}(1+N)|\eta, M\rangle .
$$

Since we discuss the problem in the space $S$ (Eq.(15)), we can multiply Eq.(23) by $1 /[(1+$ $N) \sqrt{N+1-M}]$ from left. This leads to

$$
[\sqrt{N+1-M} /(N+1)] a|\eta, M\rangle=\sqrt{1-\eta}|\eta, M\rangle
$$

The above equation shows that the NBS are nonlinear coherent states with the nonlinear function $\sqrt{N+1-M} /(N+1)$. Eq.(24) naturally reduces to Eq.(21) for $M=0$. 


\section{NONCLASSICAL PROPERTIES}

\section{A. Sub-Poissonian distribution}

The simplest way to investigate the statistical characteristics of the radiation field is to differentiate the generation function

$$
G(\lambda)=\sum_{n=0}^{\infty} P(n) \lambda^{n}=\lambda^{M}\left(\frac{\eta}{1+\lambda \eta-\lambda}\right)^{M+1}
$$

with respect to the auxiliary real number $\lambda$. Here $P(n)=\left|C_{n}(\eta, M)\right|^{2}$ is the photon distri-

bution function of the NBS. The factorial moments are defined as $F(n)=\left.\frac{d^{n} G}{d \lambda^{n}}\right|_{\lambda=1}$. From Eq.(25) we obtain the factorial moments $F(1)$ and $F(2)$ as

$$
\begin{aligned}
& F(1)=\langle N\rangle=\frac{M+1}{\eta}-1 \\
& F(2)=\left\langle N^{2}\right\rangle-\langle N\rangle=\frac{(M+2)(M+1)}{\eta^{2}}-4 \frac{M+1}{\eta}+2 .
\end{aligned}
$$

Then we can easily derive Mandel's Q parameter

$$
\begin{aligned}
Q & =\frac{\left\langle N^{2}\right\rangle-\langle N\rangle^{2}-\langle N\rangle}{\langle N\rangle} \\
& =\frac{F(2)-F^{2}(1)}{F(1)}=\frac{\eta^{2}-2(M+1) \eta+M+1}{\eta(M+1-\eta)},
\end{aligned}
$$

which measures the deviation from the Poisson distribution which corresponds to the coherent state with $Q=0$. If $Q<0(>0)$, the field is called sub(super)-Poissonian. The denominator of Eq.(28) is positive since $\eta \leq 1$, while the numerator can be positive or negative. For $M=0, Q=(1-\eta) / \eta \geq 0$. The NBS $|\eta, 0\rangle$ (geometric state) is super-Poissonian except $\eta=1$. For $M>0$, the condition for the numerator $\eta^{2}-2 \eta(M+1)+M+1<0$ is

$$
\eta>\eta_{-}=M+1-\sqrt{M(M+1)} .
$$

It can be proved that $0<\eta_{-}<1$. Thus the NBS $|\eta, M\rangle^{-}(M>0)$ is super-Poissonian when $\eta<\eta_{-}$and sub-Poissonian when $\eta>\eta_{-}$. As $M$ increases, $\eta_{-}$decreases and the sub-Poissonian range increases. 


\section{B. Squeezing effect}

Define the quadrature operators $X$ (coordinate) and $Y$ (momentum) by

$$
X=\frac{1}{2}\left(a+a^{\dagger}\right), Y=\frac{1}{2 i}\left(a-a^{\dagger}\right)
$$

Then their variances

$$
\operatorname{Var}(X)=\left\langle X^{2}\right\rangle-\langle X\rangle^{2},\left\langle\operatorname{Var}(Y)=\left\langle Y^{2}\right\rangle-\langle Y\rangle^{2}\right.
$$

obey the Heisenberg's uncertainty relation

$$
\operatorname{Var}(X) \operatorname{Var}(Y) \geq \frac{1}{16}
$$

If one of the $\operatorname{Var}(X)$ and $\operatorname{Var}(Y)$ is less than $1 / 4$, the squeezing occurs. In the present case, $\langle a\rangle$ and $\left\langle a^{2}\right\rangle$ are real. Thus, the variances of $X$ and $Y$ can be written as

$$
\begin{aligned}
& \operatorname{Var}(X)=\frac{1}{4}+\frac{1}{2}\left(\left\langle a^{\dagger} a\right\rangle+\left\langle a^{2}\right\rangle-2\langle a\rangle^{2}\right), \\
& \operatorname{Var}(Y)=\frac{1}{4}+\frac{1}{2}\left(\left\langle a^{\dagger} a\right\rangle-\left\langle a^{2}\right\rangle\right) .
\end{aligned}
$$

From Eq.(2), the expectation values $\langle a\rangle$ and $\left\langle a^{2}\right\rangle$ are obtained as

$$
\begin{aligned}
\langle a\rangle & =\eta^{M+1}(1-\eta)^{-M} \sum_{n=M}^{\infty}\left(\begin{array}{c}
n+1 \\
M
\end{array}\right)^{1 / 2}\left(\begin{array}{c}
n \\
M
\end{array}\right)^{1 / 2}(1-\eta)^{n+1 / 2}(n+1)^{1 / 2} \\
\left\langle a^{2}\right\rangle & =\eta^{M+1}(1-\eta)^{-M} \sum_{n=M}^{\infty}\left(\begin{array}{c}
n+2 \\
M
\end{array}\right)^{1 / 2}\left(\begin{array}{c}
n \\
M
\end{array}\right)^{1 / 2}(1-\eta)^{n+1}[(n+2)(n+1)]^{1 / 2}
\end{aligned}
$$

Using Eqs.(26), and (33)-(36), we can investigate the squeezing effect.

By numerical calculations, we find that the squeezing occurs in both the quadrature $X$ and $Y$. Fig. 1 gives the variance of the quadrature $X$ versus $\eta$ for different $M$. It can be seen that the range and degree of squeezing increase as $M$ increases. There exists a critical value of $M$. When $M<7$, there is no squeezing for arbitrary values of $\eta$. The squeezing also occurs in the quadrature $Y$ as shown in Fig.2. In contrary to the squeezing in the quadrature $X$, the range and degree decrease as $M$ increases. When $M$ is larger than a critical value 31, no squeezing occurs. 


\section{THE Q AND WIGNER FUNCTIONS}

If a field is prepared in a quantum state described by a density operator $\rho$, we can define the $s$-parametrized quasiprobability distribution in phase space as[27]

$$
P(\beta, s)=\frac{1}{\pi^{2}} \int d^{2} \xi C(\xi ; s) \exp \left(\beta \xi^{*}-\beta^{*} \xi\right)
$$

where the quantum characteristic function is

$$
C(\xi, s)=\operatorname{Tr}[D(\xi) \rho] \exp \left(s|\xi|^{2} / 2\right)
$$

Here $\beta=x+i y$, with $(x, y)$ being the $c$ numbers corresponding to the quadratures $(X, Y)$, and $D(\xi)=\exp \left(\xi a^{\dagger}-\xi^{*} a\right)$ is Glauber's displacement operator. It is possible to write the $s$-parametrized quasiprobability distribution as a infinite series [28]

$$
P(\beta, s)=\frac{2}{\pi} \sum_{n=0}^{\infty}(-1)^{k} \frac{(1+s)^{k}}{(1-s)^{k+1}}\langle\beta, k|\rho| \beta, k\rangle,
$$

where $|\beta, k\rangle=D(\beta)|k\rangle$ is the so-called displaced number state. The expression above is suitable for direct numerical calculations.

\section{A. Q function}

If we take $s=-1$, Eq.(39) is reduced to the familiar expression for the $Q$ function

$$
Q(\beta)=\frac{1}{\pi}\langle\beta|\rho| \beta\rangle
$$

Note that since the NBS can be viewed as an excited geometric state [see Eq.(18)], we obtain the $\mathrm{Q}$ function of the NBS as

$$
Q(\beta)=\eta^{M+1} \exp \left(-|\beta|^{2}\right)|\beta|^{2 M}\left|\sum_{n=0}^{\infty} \beta^{n}(1-\eta)^{n / 2} / \sqrt{n !}\right|^{2} / M ! .
$$

In Fig.3 we present plots of the $Q$ function of a NBS for different values of $\eta$ and $M=5$. We

can clearly see the deformation of the $Q$ function. When $\eta=1$, the $Q$ function representing the number state $|M=5\rangle$ is formed[Fig3.(d)] as expected. From the $Q$ function we can also study the squeezing effects by examining the deformation of their contours. Fig. 4 is the 
contour plot of the $Q$ functions of two particular NBSs, with (a) $\eta=0.2, M=5$ and (b) $\eta=0.85, M=50$. We can clearly see the compression along the $y$ and $x$ direction, which corresponds to squeezing in the $Y$ quadrature of the first NBS and in the $X$ quadrature of the second.

\section{B. Wigner function}

By taking $s=0$ in Eq.(39), we obtain a series representation for the Wigner function

$$
W(\beta)=\frac{2}{\pi} \sum_{k=0}^{\infty}(-1)^{k}\langle\beta, k|\rho| \beta, k\rangle .
$$

Now we insert Eq.(1) into the above equation, which yields

$$
W(\beta)=\frac{2}{\pi} \sum_{k=0}^{\infty}\left|\sum_{n=M}^{\infty} C_{n}(\eta, M) \chi_{n k}(\beta)\right|^{2} .
$$

In the expression above, the matrix elements $\chi_{n k}(\beta)=\langle n|D(\beta)| k\rangle$ are given by[29]

$$
\chi_{n k}(\beta)=\beta^{n}\left(-\beta^{*}\right)^{k} \exp \left(-|\beta|^{2} / 2\right)_{2} F_{0}\left(-n,-k ;|\beta|^{-2}\right) / \sqrt{n ! k !},
$$

where ${ }_{2} F_{0}(\alpha, \beta ; z)$ are the generalized hypergeometric functions[30]. The present form of $\chi_{n k}(\beta)$ is convenient for numerical calculations.

The Wigner function can be used to trace the nonclassical behaviors of quantum states. It is known that the negativity of the Wigner function is a sufficient but not necessary condition for having nonclassical effects. In Fig.5 we give plots of the Wigner function of a NBS by numerical calculations of Eq.(43) for different values of $\eta$ and $M=1$. As in Fig.5(a), the negative part of the Wigner function is already noticeable for $\eta=0.3$. For

$\eta=0.5[$ Fig.5(b)], the negative part is pronounced. In fig5(c), for $\eta=0.9$, the negative part is even larger, and finally, in Fig.7(d), we have the full Wigner function of a number state $|1\rangle(\eta=1)$. The Wigner function becomes more and more negative as $\eta$ increases. 


\section{GENERATION OF THE NEW NBS}

Let us discuss the dynamical generation of the NBS. We consider two different methods. The first is quite straightforward in concept from the displacement operator formalism(Eq.(13)) but might not be very easy to achieve experimentally. The Hamiltonian is given by

$$
\begin{aligned}
H & =H_{0}+i \chi\left[\sqrt{\hat{N}-M} a^{\dagger} \exp (-i \omega t)-a \sqrt{\hat{N}-M} \exp (i \omega t)\right] \\
H_{0} & =\omega a^{\dagger} a
\end{aligned}
$$

The constant $\chi$ is the coupling strength. The coupling is of intensity-dependent type and is similar to those in some intensity-dependent Jaynes-Cummings models [31-34]. The unitary time evolution operator in the interaction picture is

$$
U(t)=\exp \left[\chi t\left(\sqrt{\hat{N}-M} a^{\dagger}-a \sqrt{\hat{N}-M}\right)\right] .
$$

Supposing the system is initially prepared in the number state $|M\rangle$, we find the system at time $t$ is the NBS

$$
U(t)|M\rangle=\left|1-\tanh ^{2}(\chi t), M\right\rangle .
$$

The second method of the generation of the NBS is based on the fact that the NBS is the excited geometric state. The geometric state can be prepared in the non-degenerate threewave interaction system[35]. We can also generate the geometric state by the non-degenerate parametric amplifier described by the two-mode Hamiltonian[36]

$$
\begin{aligned}
\tilde{H} & =\tilde{H}_{0}+i \chi\left[a_{1}^{\dagger} a_{2}^{\dagger} \exp (-2 i \omega t)-a_{1} a_{2} \exp (2 i \omega t)\right], \\
\tilde{H}_{0} & =\omega_{1} a_{1}^{\dagger} a_{1}+\omega_{2} a_{2}^{\dagger} a_{2},
\end{aligned}
$$

where $a_{1}$ and $\omega_{1}\left(a_{2}\right.$ and $\left.\omega_{2}\right)$ are the annihilation operator and frequency for the signal(idler) mode. Frequencies $\omega_{1}$ and $\omega_{2}$ sum to the pump frequency, $2 \omega=\omega_{1}+\omega_{2}$. The coupling constant $\chi$ is proportional to the second-order susceptibility of the medium and to the amplitude of the pump. The unitary time evolution operator in the interaction picture is

$$
\tilde{U}(t)=\exp \left[\chi t\left(a_{1}^{\dagger} a_{2}^{\dagger}-a_{1} a_{2}\right)\right]
$$


Suppose that the system is initially prepared in the state $|0,0\rangle=|0\rangle_{1} \otimes|0\rangle_{2}$. Then at any time $t$ the system is in the state

$$
|\eta\rangle_{t m}=\tilde{U}(t)|0,0\rangle=\eta^{1 / 2} \sum_{n=0}^{\infty}(1-\eta)^{n / 2}|n, n\rangle
$$

which is the two-mode geometric state in comparison with Eq.(19). Here $\eta=1-\tanh ^{2}(\chi t)$.

Once the two-mode geometric state is prepared, one can generate two-mode NBS by the following procedure in analogy to that proposed by Agawal and Tara[22]. Consider the passage of a two-level excited atom through a cavity. Let the initial state of the atom-field system be $|\eta\rangle_{t m} \otimes|e\rangle$, where $|e\rangle$ is the atomic excited state. The interaction Hamiltonian has the form[37]

$$
\bar{H}=\hbar\left(g S^{+} a_{1}+g^{*} S^{-} a_{1}^{\dagger}\right)
$$

where $S^{ \pm}$are the psedospin operators of the atom and $g$ is the coupling constant. Since $g$ is generally small, the state at time $t$ can be approximated by

$$
|\psi(t)\rangle \approx|\eta\rangle_{t m} \otimes|e\rangle-i g^{*} t a_{1}^{\dagger}|\eta\rangle_{t m} \otimes|g\rangle
$$

which is valid for interaction times $g t<<1$. From the above equation we observe that, if the atom is detected to be in the ground state $|g\rangle$, then the state of the field is reduced to $a_{1}^{\dagger}|\eta\rangle_{t m}$. An extension of the above arguments to the multiphoton processes would imply that the state $a_{1}^{\dagger M}|\eta\rangle_{t m}$ can be produced in multiphoton processes. For the multiphoton processes, the Hamiltonian (Eq.(51)) is replaced by a new Hamiltonian with $a_{1} \rightarrow a_{1}^{M}$. Thus, the above procedure for a multiphoton process will result in the state

$$
|\eta, M\rangle_{t m}=\eta^{(M+1) / 2} \sum_{n=0}^{\infty}\left(\begin{array}{c}
M+n \\
M
\end{array}\right)^{1 / 2}(1-\eta)^{n / 2}|M+n, n\rangle .
$$

The above normalized state is just the two-mode NBS. In a short summary two methods are proposed to generate the NBS. 


\section{CONCLUSIONS}

We have investigated the NBS induced recently by Barnett and found the ladder operator formalism and displacement operator formalism of the NBS. The algebra involved is SU(1,1) Lie algebra via the generalized Holstein-Primakoff realization. We found that the NBS are essentially Peremolov's coherent states.

As excited quantum states, the NBSs are excited geometric states. As intermediate states, they interpolate between the number and geometric states. According to the definition of the nonlinear coherent states, we find that the NBSs are nonlinear coherent states.

The NBS can be sub-Poissonian or super-Poissonian. There exists a critical point $\eta_{-}$. The NBS is sub-Poissonian when $\eta>\eta_{-}$and super-Poissonian when $\eta<\eta_{-}$. The squeezing occurs in both the quadrature $X$ and $Y$ with two critical values of $M=7$ and 31, respectively. There is no squeezing occurs in the quadrature $X$ for $M<7$ and in the quadrature $Y$ for $M>31$ for arbitrary values of $\eta$. The $Q$ and Wigner functions are studied numerically. They show that the NBSs have prominent nonclassical properties. We have proposed two methods of generation of the new NBS.

In addition, the remarkable properties of the new NBS seem to suggest that it deserves further attention from both theoretical and application sides of quantum optics.

Acknowledgment: One of the authors(Wang) thanks for the discussions with Prof.

H.C.Fu and help of Prof. C.P.Sun. The work is partially supported by the National Science Foundation of China with grant number:19875008. 


\section{REFERENCES}

[1] D.Stoler, B.E.A.Saleh and M.C.Teich, Opt.Acta. 32,345(1985)

[2] G.Dottoli, J.Gallardo and A.Torre , J.Opt.Soc.Am. B 2, 185(1987)

[3] C.T.Lee , Phys.Rev. A 31, 1213(1985)

[4] A.Vidiella-Barranco and J.Roversi, Phys.Rev. A 50 ,5233(1994)

[5] H.Y.Fan and S.J.Jing ,Phys.Rev. A 50,1909(1994)

[6] H.C.Fu and R.Sasaki ,J.Phys.A:Math.Gen. 29 5637(1996)

[7] A.Joshi and S.V.Lawande ,Opt.Commun. 70 21(1989)

[8] K.Matsuo,Phys.Rev. A 41, 519(1990)

[9] A.Joshi and S.V.Lawande ,J.Mod.Opt. 38,2009(1991)

[10] G.S.Agarwal, Phys.Rev. A 45, 1787(1992)

[11] H.C.Fu and R.Sasaki ,J.Phys.Soc.Japan 66,1989(1997)

[12] H.C.Fu and R.Sasaki, J.Math.Phys. 38, 2154(1997)

[13] H.Y.Fan and N.L.Liu, Phys.Lett. A 250, 88(1998)

[14] S.M.Barnett ,J.Mod.Opt. 45, 2201(1998)

[15] E.Jakeman and T.J.Shephard ,J.Phys.A:Math.Gen. 17, L745(1984)

[16] J.H.Shapiro and S.R.Shephard , Phys.Rev.Lett. 62, 2377(1991)

[17] J.H.Shapiro and S.R.Shephard, Phys.Rev. A 43 3795(1991)

[18] E.C.Lerner, H.W.Huang and G.E.Walters ,J.Math.Phys. 11 1679(1970).

[19] M.J.W.Hall, J.Mod.Opt. 40 ,809(1993)

[20] A.-S.F.Obada, S.S.Hassan, R.R.Puri and M.S.Abdalla Phys.Rev. A 48,3174(1993) 
[21] A.-S.F.Obada, O.M.Yassin and S.M.Barnett, J.Mod.Opt. 44 149(1997)

[22] G.S.Agarwal and K.Tara, Phys.Rev. A 43,492(1991).

[23] L.Susskind and J.Glogower, Physics 1,49(1964)

[24] R.L.de Matos Filho and W.Vogel, Phys.Rev.A 54, 4560(1996)

[25] O.V.Man'ko, Phys.Lett. A228, 29(1997).

[26] X.G.Wang and H.C.Fu, Int.J.Mod.Phys.B. 13, 617(1999).

[27] K.E.Cahill and R.J.Glauber, Phys.Rev. 177, 1857(1969).

[28] H.Moya-Cessa and P.L.Knight, Phys.Rev. A 48, 2479 (1993).

[29] X.G.Wang, Commun.Theor.Phys., to be published.

[30] Yu.V.Geronimus and M.Yu.Tseytlin, Table of Integrals, Series and Products, edited by Alan Jeffrey (Academic, New York, 1980).

[31] B.Buck and C.V.Sukumar, Phys.Lett.A 81,132(1981).

[32] J.Crnugelj, M.Martinis and V.Mikuta-Martinis, Phys.Lett.A 188, 347(1994).

[33] D.Bonatosos and C.Daskaloyannis and G.A.Lalasissis, Phys.Rev.A 47, 3448(1993).

[34] X.G.Wang, Chinese Science Bulletin 41,17(1996).

[35] G.M.D'Ariano, M.G.A.Paris and M.F.Sacchi, quant-ph/9803015.

[36] D.F.Walls and G.J.Milburn, Quantum Optics (Springer-Verlag, 1994).

[37] E.T.Jaynes and F.W.Cummings, Proc.IEEE 51,89(1963). 


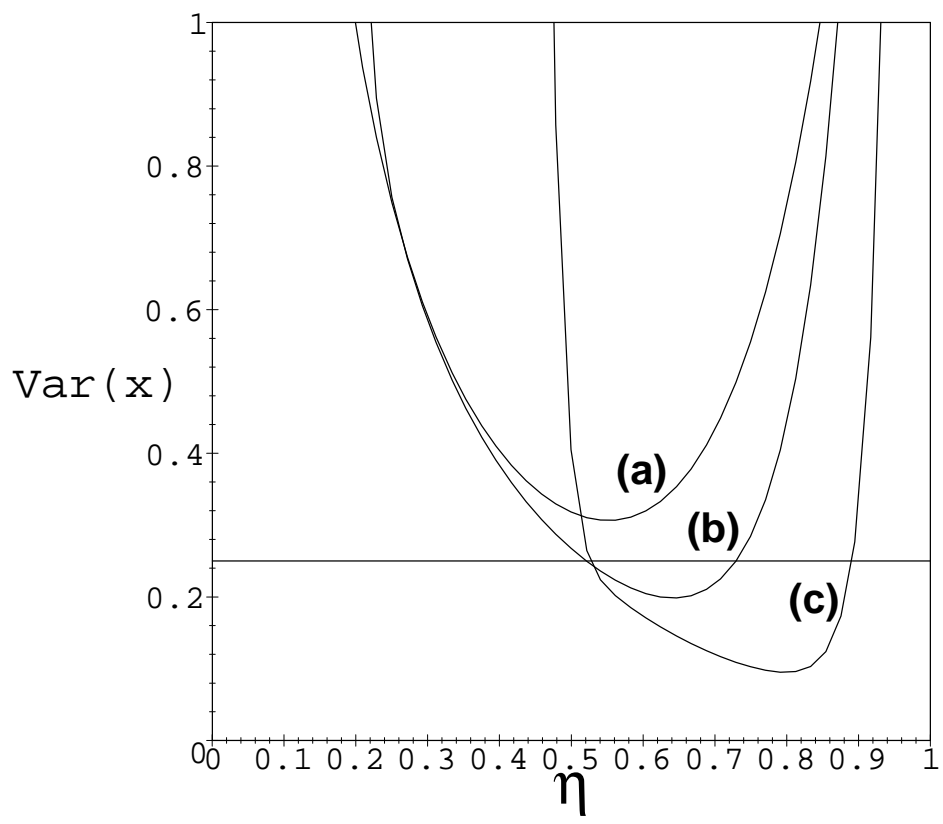

FIG. 1. The variance of the quatrature $X$ as a function $\eta$ for different values of $M$ : (a) $M=5$, (b) $M=10$, and (c) $M=35$. 


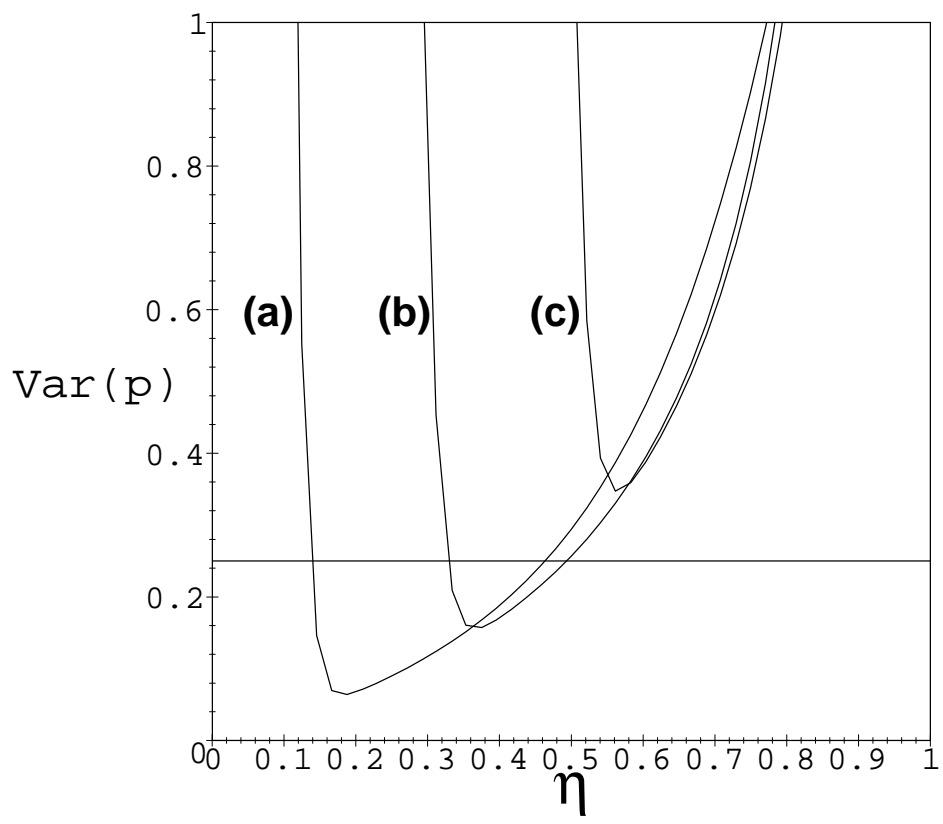

Fig.2. The variance of the quatrature $Y$ as a function $\eta$ for different values of $M$ : (a) $M=5$, (b) $M=20$, and (c) $M=40$. 

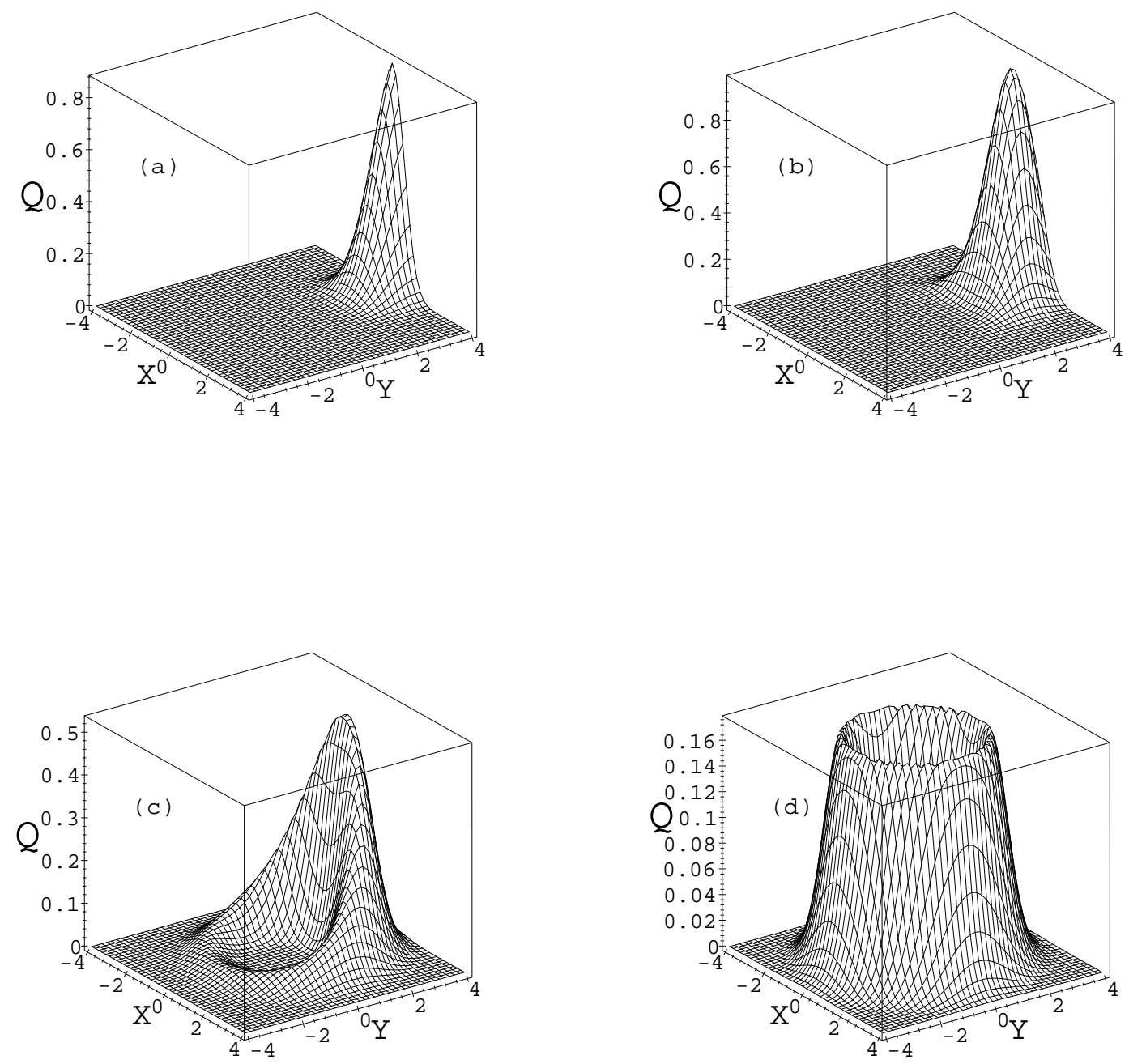

Fig.3. Q function of a NBS for four values of $\eta$ : (a) $\eta=0.3$, (b) $\eta=0.5,(\mathrm{c}) \eta=0.9$, and (d) $\eta=1$. In all cases $M=5$. 


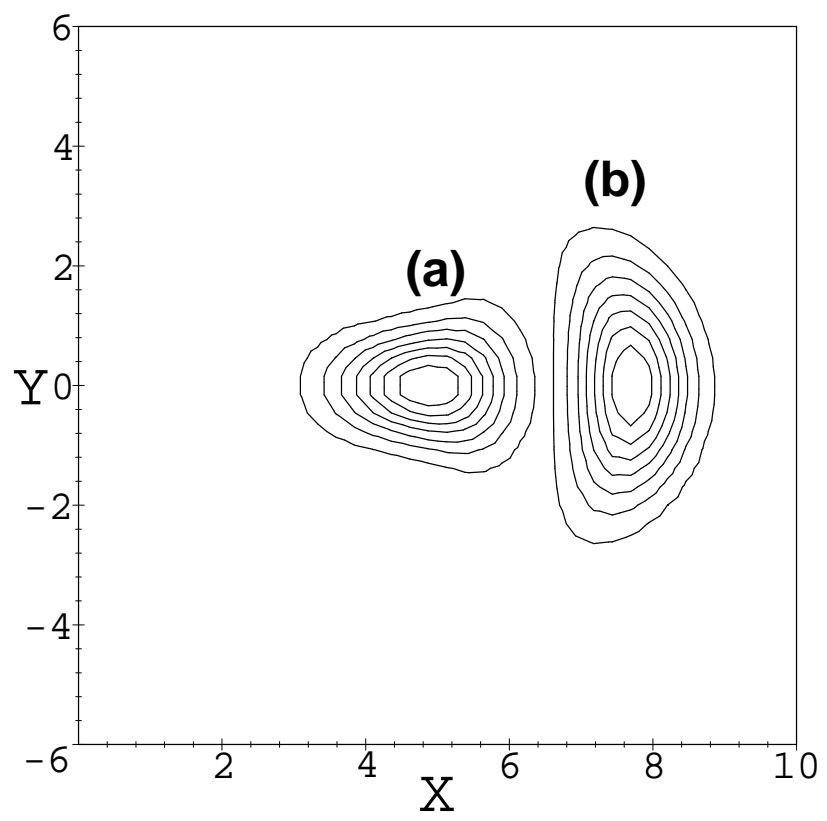

Fig.4 Contours of the Q function of two NBSs with (a) $\eta=0.2, M=5$, and (b) $\eta=0.85, M=50$ 

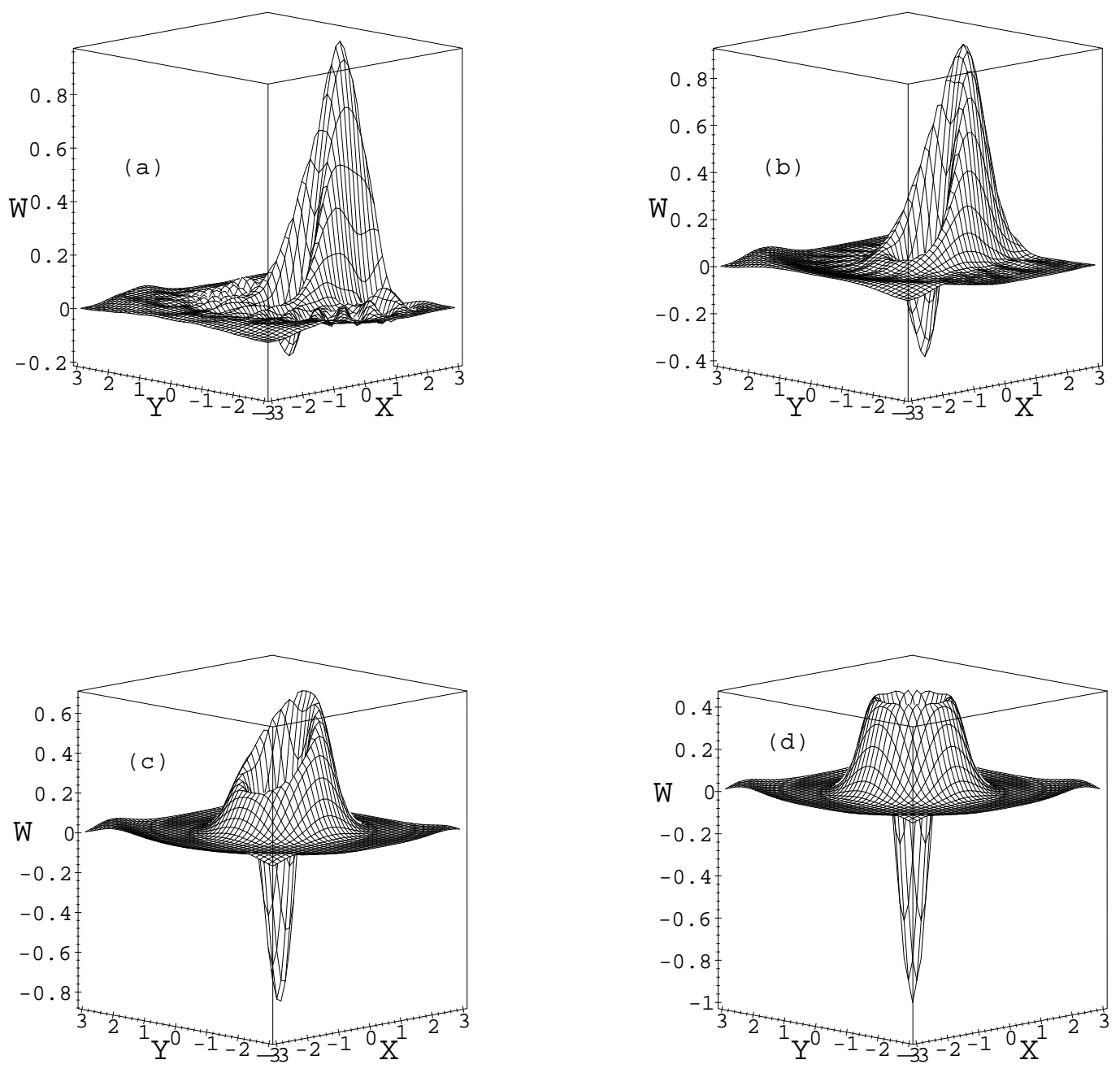

Fig.5. Wigner function of a NBS for four values of $\eta$ : (a) $\eta=0.3$, (b) $\eta=0.5$, (c) $\eta=0.9$, and (d) $\eta=1$. In all cases $M=1$. 